\title{
Evidence for Breathing of a Class I Fusion Protein at the Cell Surface
}

\author{
Morgan Gilman¹, Polina Furmanova², Gabriel Pasqual², Jennifer Lum³, Jay Wadia, \\ Anthony Williamson', Angelique van 't Wout ${ }^{3}$, Jerome Custer', \\ Johannes P M Langedijk², Jason McLellan ${ }^{4}$ \\ ${ }^{1}$ Department of Biochemistry, Geisel School of Medicine at Dartmouth, Hanover, New Hampshire, USA \\ ${ }^{2}$ Janssen Infectious Diseases and Vaccines, Leiden, The Netherlands \\ ${ }^{3}$ Janssen Prevention Center, Janssen Vaccines \& Prevention B.V., Leiden, The Netherlands \\ ${ }^{4}$ Department of Molecular Biosciences, University of Texas at Austin, Austin, Texas, USA
}

Respiratory syncytial virus (RSV) causes a substantial disease burden in infants and the elderly worldwide. Of the two glycoproteins present on the viral surface-the fusion protein $(F)$ and the attachment protein $(\mathrm{G})$-only $\mathrm{F}$ is absolutely required for infection. RSV F is a class I fusion protein, which exists in a metastable conformation (prefusion $\mathrm{F}$ ) that undergoes dramatic conformational changes to mediate viral entry. We isolated an antibody, called CR9501, that potently neutralizes RSV and prevents the rearrangements of prefusion $\mathrm{F}$ required for membrane fusion. Surprisingly, an RSV F-CR9501 complex crystallized as a monomer, despite being fused to a trimerization motif. Additional structural and biochemical studies demonstrated that CR9501 accelerates the disassembly of prefusion $\mathrm{F}$ trimers into component monomers and exhibits distinct competition profiles on trimeric and monomeric $F$. We utilized the unique properties of CR9501 to demonstrate that full-length $\mathrm{F}$ trimers sample a monomeric conformation on the surface of transfected cells. In order to reduce flexibility and facilitate crystal packing, a monomeric variant of prefusion $\mathrm{F}$ was complexed with CR9501 and crystallized, resulting in a $3.3 \AA$ crystal structure of the antibody-F complex. Analysis of this structure, as well as over 30 previously determined prefusion $\mathrm{F}$ structures, revealed a breathing motion that may precede the opening of $F$ at the cell surface. Collectively, these findings suggest that the prefusion $\mathrm{F}$ trimer breathes on the surface of cells and have implications for understanding the role of oligomerization in class I-mediated membrane fusion. 